\title{
Exosomes derived from bone marrow mesenchymal stem cells overexpressing microRNA-25 protect spinal cords against transient ischemia
}

\author{
Linlin Zhao, MD, ${ }^{\mathrm{a}, \mathrm{b}}$ Xiaojing Jiang, MD, PhD, ${ }^{\mathrm{c}}$ Jiang Shi, MD, ${ }^{\mathrm{a}}$ Shilun Gao, MD, ${ }^{\mathrm{a}}$ Yubao Zhu, MD,
} Tianxiang Gu, MD, PhD, ${ }^{\mathrm{a}}$ and Enyi Shi, $\mathrm{MD}, \mathrm{PhD}^{\mathrm{a}}$

\section{ABSTRACT}

Objective: We investigated the neuroprotection of exosomes derived from bone marrow mesenchymal stem cells overexpressing microRNA- 25 on ischemic spinal cords.

\begin{abstract}
Methods: Cultured mesenchymal stem cells were transfected with lentivirus vectors containing pre-microRNA-25 or control vectors. Exosomes were isolated and harvested by centrifugation. Spinal cord ischemia was induced in rats by crossclamping the descending aorta just distal to the left subclavian artery for 15 minutes. Exosomes from mesenchymal stem cells, mesenchymal stem cells transfected with control vector, or pre-microRNA-25 were administered by intrathecal injection before ischemia. Hind-limb motor function was assessed with the motor deficit index. Contents of interleukin- $1 \beta$, tumor necrosis factor- $\alpha$, malondialdehyde, and superoxide dismutase activity were measured using commercial kits. Expressions of NADPH oxidase 2, NADPH oxidase 4, and microRNA-25 were detected by Western blot and quantitative reverse transcription polymerase chain reaction. Lumbar spinal cords were harvested for histologic examination.
\end{abstract}

Results: Transfection of pre-microRNA-25 significantly enhanced microRNA25 levels in mesenchymal stem cells and their exosomes $(P<.001)$. All exosome-pretreating groups exhibited lower levels of interleukin- $1 \beta$ and tumor necrosis factor- $\alpha(P<.001)$, more intact motor neurons $(P<.001)$, and lower motor deficit index scores $(P<.005)$ than those of controls. Compared with exosomes, microRNA-25-enriched exosomes markedly enhanced microRNA-25 level $(P<.001)$, inhibited NADPH oxidase 4 expression $(P=.012)$, but not NADPH oxidase 2 expression, decreased malondialdehyde content $(P=.022)$, increased superoxide dismutase activity $(P<.001)$ in spinal cords, and had additional neuroprotective effects as evidenced by lower motor deficit index scores $(P<.005)$ and more survival neurons $(P=.002)$.

Conclusions: The neuroprotection of exosomes from mesenchymal stem cells on ischemic spinal cords can be enhanced by genetic modification of the exosomes to contain elevated microRNA-25. (J Thorac Cardiovasc Surg 2019;157:508-17)

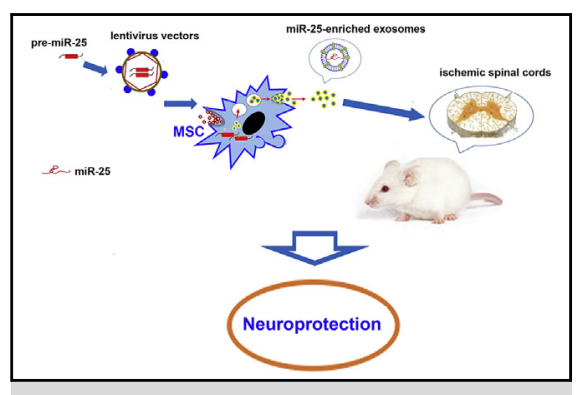

miR-25-enriched exosomes confer neuroprotection on spinal cords against ischemia-reperfusion injury.

\section{Central Message}

Exosomes derived from MSCs conduct neuroprotection on ischemic spinal cords, and the neuroprotection can be enhanced by genetic modification of the exosomes to contain elevated miR-25.

\section{Perspective}

The neuroprotection of MSC-derived exosomes on ischemic spinal cords was improved by tailoring the exosomes to encapsulate miR25 in the current study. By extension, ongoing studies to investigate genetic modification of MSC-derived exosomes may provide a novel cell-free therapeutic strategy for prevention of neurologic injury after thoracic aneurysm surgery.

See Editorial Commentary page 518.
From the Departments of ${ }^{\mathrm{a}}$ Cardiac Surgery and ${ }^{\mathrm{c}}$ Anesthesiology, First Affiliated Hospital, China Medical University, Shenyang, China; and ${ }^{\mathrm{b}}$ Department of Cardiac Surgery, the People's Hospital of Liaoning Province, Shenyang, China.

This work was supported by Grant No. 81471267 from the National Natural Science Foundation of China, Beijing, P. R. China.

T.G. and E.S. contributed equally to this work.

Received for publication April 4, 2018; revisions received July 10, 2018; accepted for publication July 11, 2018; available ahead of print Sept 14, 2018.

\footnotetext{
Address for reprints: Enyi Shi, MD, PhD, Department of Cardiac Surgery, First Affiliated Hospital, China Medical University, Shenyang, P. R. China, 110001 (E-mail: shienyi2002@hotmail.com). 0022-5223/\$36.00

Copyright (ㄷ 2018 by The American Association for Thoracic Surgery

https://doi.org/10.1016/j.jtcvs.2018.07.095
} 

Abbreviations and Acronyms
$\mathrm{IL} \quad=$ interleukin
$\mathrm{MDA}=$ malondialdehyde
MDI $=$ motor deficit index
$\operatorname{miR}=$ microRNA
MSC $=$ mesenchymal stem cell
NOX $=$ NADPH oxidase
PBS $=$ phosphate-buffered saline
ROS $=$ reactive oxygen species
SOD $=$ superoxide dismutase
$\mathrm{TNF}=$ tumor necrosis factor

Scanning this QR code will take you to the article title page to access supplementary information.

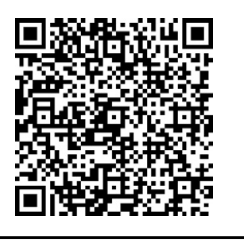

Paraplegia induced by transient or permanent spinal cord ischemia is one of the most devastating complications with a high incidence after open and endovascular thoracoabdominal aortic aneurysm repair. Regardless of the developments of surgical techniques and perioperative strategies, spinal cord ischemia is still understood incompletely and not entirely preventable. ${ }^{1}$

Exosomes are an endosome-derived subtype of membrane vesicles, approximately 30 to $100 \mathrm{~nm}$ in diameter, and are released into extracellular fluids by almost every cell type. ${ }^{2}$ Exosomes carry a wide range of cargo, including messenger RNA, microRNA (miR), cytoskeletal elements, proteins, enzymes, molecular chaperones, and signaling molecules and play essential roles in intercellular communication. Such cargoes can be shipped to target cells under physiologic and pathophysiologic conditions that induce expressional and functional changes. ${ }^{2}$ Exosomes from different cell types have attracted much interest recently with regard to their role in the nervous system. Endothelial cell-derived exosomes have been indicated to directly protect nerve cells against ischemia-reperfusion injury. ${ }^{3}$ Astrocyte-derived exosomes are shown to improve the survival of neurons under hypoxic and ischemic conditions. ${ }^{4}$

Mesenchymal stem cells (MSCs) can promote angiogenesis, neurogenesis, and white matter remodeling in the injured brain by secreting factors to trigger the signaling pathways that are involved in brain repair. The strong paracrine capacity of MSCs, but not their differentiation capacity and cell replacement, is the principal mechanism of their therapeutic action. ${ }^{5}$ Our previous studies show that MSCs can attenuate neurologic injuries of spinal cord ischemiareperfusion injury, ${ }^{6}$ and hepatocyte growth factor genemodified MSCs can further improve the neuroprotective effect. ${ }^{7}$ Compared with other cell types, cultured MSCs secrete a large quantity of exosomes. ${ }^{8}$ Systemic administration of exosomes released from MSCs promotes functional recovery and neurovascular plasticity after stroke in rats. Via exosomes, MSCs transfer their therapeutic factors, especially miR, to recipient cells, and therein alter gene expression and thereby promote therapeutic response. ${ }^{10}$ MiR-133b transferred by exosomes from MSCs contributes to neurite remodeling and functional recovery after stroke. ${ }^{11}$ Improved neurologic outcomes from MSCderived exosome studies are comparable to the therapeutic effect observed with MSC therapy. ${ }^{2}$ Furthermore, the release and the content of exosomes can be modified by genetic manipulation for its source cells. Adipose-derived MSCs engineered to overexpress miR-181-5p are able to selectively transfer miR-181-5p to target cells. ${ }^{12}$

miRs are 21- to 23-nucleotide nonprotein-coding RNA molecules that act as negative regulators of gene expression by degrading the target messenger RNA or arresting its translation. ${ }^{13}$ miR-based strategies have recently emerged as a promising therapeutic approach for ischemic stroke in animals. ${ }^{14}$ In our previous reports, regulation of miRs is indicated to improve functional recovery and attenuate neurologic injury after spinal cord ischemia in rats, ${ }^{15,16}$ which expands the understanding of miRs as neuroprotective molecular targets against ischemic injury of spinal cords.

miR-25 has been reported to be a major miR marker in neural cells and is strongly expressed in ischemic brain tissues. ${ }^{17}$ Repetitive transcranial magnetic stimulation promotes neural stem cell proliferation via upregulation of miR-25 in a rat model of focal cerebral ischemia. ${ }^{18}$ Upregulation of miR-25 also inhibits human glioblastoma cell apoptosis induced by oxygen-glucose deprivation through downregulating Fas/FasL. ${ }^{19}$ Moreover, miR-25 is involved in regulation of oxidative stress through its target protein NADPH oxidase (NOX) 4. Hypercholesterolemia enhances NOX4 expression by downregulating miR-25 and consequently increases oxidative/nitrative stress in the heart. ${ }^{20}$ The NOX activity is associated with miR-25-mediated NOX4 expression in experimental diabetic nephropathy, and miR-25 negatively regulates NOX4 expression by directly targeting the 3 '-UTR. ${ }^{21}$ However, little is known about the role and underlying mechanisms of miR-25 in ischemia-reperfusion injury of spinal cords.

In the current study, we delivered miR-25 to spinal cords by exosomes derived from MSCs that were genetically modified to overexpress miR-25. We highlight the neuroprotective effect of miR-25-modified exosomes on spinal cords in the setting of transient ischemia. 


\section{MATERIALS AND METHODS Animals}

Male Sprague Dawley rats weighing approximately $250 \mathrm{~g}$ were enrolled in the present study. The animal protocol was approved by the Ethics Review Committee for Animal Experimentation of China Medical University (Shenyang, People's Republic of China).

\section{Mesenchymal Stem Cell Culture and Transfection}

Sprague Dawley rats' bone marrow MSCs were purchased from Cyagen Biosciences Inc (Guangzhou, China) and cultured in rat MSC basal media supplemented with $10 \%$ MSC-qualified fetal bovine serum, $1 \%$ penicillinstreptomycin, and $1 \%$ glutamine. Cells were passaged when they were approximately $90 \%$ confluent and were used at passage 3 .

Rat pre-miR-25 lentivirus gene transfer vectors were constructed by Genechem (Shanghai, China), and lentivirus gene transfer vectors without pre-miR-25 were used as controls. $1 \times 10^{6}$ MSCs were seeded in $10 \mathrm{~mL}$ of Dulbecco's Modified Eagle Medium supplemented with $10 \%$ fetal bovine serum overnight and were subsequently transfected with $100 \mathrm{nM}$ pre-miR25 or control vector at a multiplicity of infection of 10 for 24 hours. After infection, the envelope suspension was changed to normal culture medium, and the infected MSCs were continuously cultured for 48 hours.

\section{Exosome Extraction and Identification}

At 48 hours after transfection, cultured MSCs were harvested for quantitative real-time polymerase chain reaction. The exosome isolation was performed at $4{ }^{\circ} \mathrm{C}$, as previously described. ${ }^{11}$ Briefly, the supernatants collected from cultured MSCs were first filtered to remove large debris and dead cells. Small cell debris was removed by centrifugation at $10,000 \times \mathrm{g}$ for 30 minutes, and then the resulting supernatants were further centrifuged at $100,000 \times \mathrm{g}$ for 3 hours. The pellet was resuspended in phosphate-buffered saline (PBS). The sizes of the pellets were measured by nanoparticle tracking analysis. Expressions of CD9, CD63, and CD81, as markers of exosomes from MSCs, were detected by Western blot. The presence of exosome was also verified by electron microscopy.

\section{Spinal Cord Ischemia}

Spinal cord ischemia was induced by crossclamping the descending aorta just distal to the left subclavian artery for 15 minutes as described previously (Video 1). ${ }^{15,16}$

\section{Intrathecal Injection}

Exosomes or PBS were administered in vivo by means of intrathecal injection, as reported previously. ${ }^{15,16}$ Animals were included in the following protocol only if they had normal hind-limb motor function before the surgical procedure.

\section{Experimental Protocol}

Male Sprague Dawley rats were divided into 5 groups randomly. All rats except sham animals received a 15-minute spinal cord ischemia. (1) Sham group $(\mathrm{n}=11)$ : Rats received only the surgical procedure without spinal cord ischemia. (2) Control group $(\mathrm{n}=11)$ : PBS $(10 \mu \mathrm{L})$ was injected intrathecally into each rat 1 day before spinal cord ischemia. (3) Exo group $(\mathrm{n}=12)$ : Exosomes derived from MSCs $(10 \mu \mathrm{L}, 2 \mu \mathrm{g} / \mu \mathrm{L}$ in PBS $)$ were injected intrathecally into each rat 1 day before spinal cord ischemia. (4) Vector-Exo group $(\mathrm{n}=10)$ : Exosomes derived from MSCs transfected with control vector ( $10 \mu \mathrm{L}, 2 \mu \mathrm{g} / \mu \mathrm{L}$ in PBS) were injected intrathecally into each rat 1 day before spinal cord ischemia. (5) miR-25-Exo group $(\mathrm{n}=11)$ : Exosomes derived from MSCs transfected with lentivirus vector containing pre-miR-25 (10 $\mu \mathrm{L}, 2 \mu \mathrm{g} / \mu \mathrm{L}$ in PBS) were injected intrathecally into each rat 1 day before spinal cord ischemia.

In a parallel series of experiments, spinal cords were also collected 6 hours after reperfusion from the 5 groups ( $n=5$ per group) to evaluate

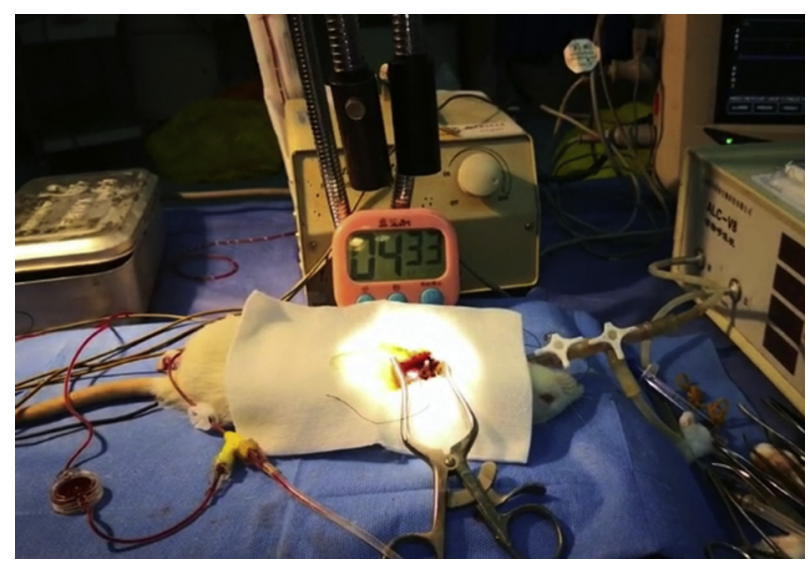

VIDEO 1. Spinal cord ischemia was induced in rats by crossclamping the descending aorta just distal to the left subclavian artery, and exosomes were administered by intrathecal injection. Transient spinal cord ischemia induced complete paraplegia in a control rat. Video available at: https:// www.jtcvs.org/article/S0022-5223(18)32136-6/fulltext.

contents of interleukin (IL)- $1 \beta$, tumor necrosis factor (TNF) and malondialdehyde (MDA), and superoxide dismutase (SOD) activity in spinal cords. Expressions of miR-25, NOX2, and NOX4 in spinal cords were also measured.

\section{Neurologic Assessment}

Hind-limb motor function was assessed before spinal cord ischemia and at 24 hours, 48 hours, and 7 days after reperfusion via motor deficit index (MDI) score (quantified by ambulation and the placing/stepping reflex $)^{15,16}$ by investigators who were blinded to group information.

\section{Enzyme-Linked Immunosorbent Assay}

Contents of IL- $1 \beta$ and TNF- $\alpha$ in spinal cords were determined using enzyme-linked immunosorbent assay kits (Bioyotime, Shanghai, China) according to the manufacturer's instructions.

\section{Malondialdehyde and Superoxide Dismutase Measurement}

MDA level and SOD activity in spinal cords were determined using commercial assay kits (Jiancheng Bioengineering Institute, Nanjing, China) according to the manufacturer's protocol.

\section{Histologic Study}

Rats were killed 7 days after reperfusion. Paraffin-embedded sections $(4 \mu \mathrm{m})$ of lumbar spinal cords (L4-L6) were stained with Nissl dye. Gray-matter damage was assessed by counting the number of normal motor neurons in the ventral part of the gray matter as described previously. ${ }^{15,16}$

\section{Quantitative Real-Time Polymerase Chain Reaction}

Total RNA of spinal cords or cultured MSCs was isolated with Trizol reagent (Invitrogen, Carlsbad, Calif) according to the manufacturer's instruction. Quantitative real-time polymerase chain reaction was performed according to a standard protocol with the Step One Plus system (Applied Biosystems, Foster City, Calif). U6 served as an internal control. Changes in expression were determined by the $2^{-\Delta \Delta \mathrm{CT}}$ method.

\section{Western Blot}

CD9, CD81, and CD63 in exosomes and NOX2 and NOX4 in spinal cords were analyzed by sodium dodecyl sulfate polyacrylamide gel 


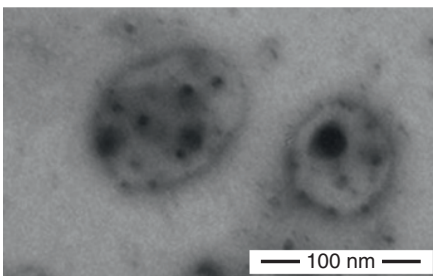

A

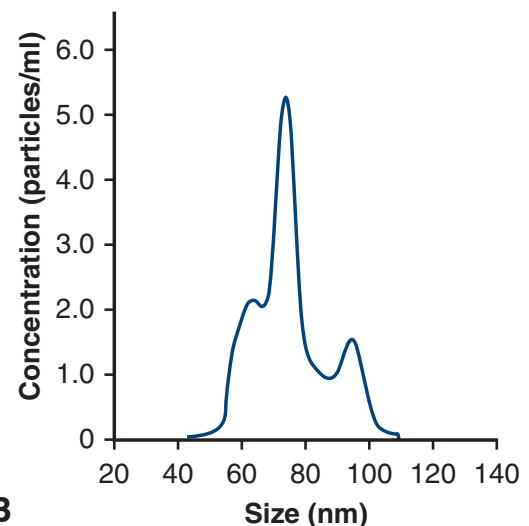

B

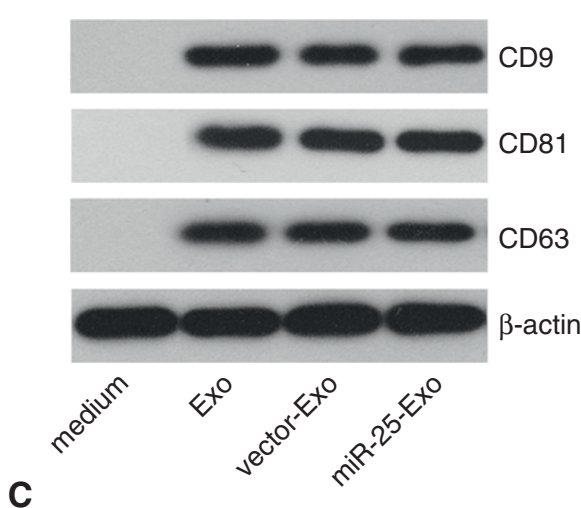

FIGURE 1. Exosome characterization. A, Electron microscopic image of miR-25-enriched exosomes. B, The result of nanoparticle tracking analysis of miR-25-enriched exosomes. C, Western blots showing expressions of CD9, CD81, and CD63 in exosomes. Exo, Exosomes derived from MSCs; vector-Exo, exosomes derived from MSCs transfected with control vectors; miR-25-Exo, miR-25-enriched exosomes derived from MSCs transfected with pre-miR-25.

electrophoresis and immunoblotted with anti-CD9, anti-CD63, anti-CD81, anti-NOX2, and anti-NOX4 antibodies (Abcam, Cambridge, Mass). The protein expressions were analyzed with NIH Image (Research Services Branch, National Institutes of Health, Bethesda, Md) and quantified as a relative fold to the sham group after normalization with $\beta$-actin.

\section{Statistical Analysis}

Values of miR-25, NOX-2, NOX-4, IL-1 $\beta$, TNF- $\alpha$, MDA, and SOD were analyzed by 1-way analysis of variance followed by Bonferroni correction for post hoc testing. MDI scores and numbers of neurons were analyzed by Kruskal-Wallis test with Bonferroni correction $(P<.005$ before Bonferroni correction was considered statistically significant). Statistical analysis was performed with SPSS, version 19.0 (IBM-SPSS Inc, Armonk, NY).

\section{RESULTS}

\section{Exosome Characterization}

Exosomes derived from MSCs overexpressing miR-25 were membrane vesicles as shown by transmission electron microscopy (Figure 1,A) and were 50 to $100 \mathrm{~nm}$ in diameter (Figure 1, $B$ ). Expressions of CD9, CD63, and CD81 in exosomes were confirmed by Western blotting (Figure 1,C).

\section{MicroRNA-25 Levels in Mesenchymal Stem Cells Transfected With Pre-MicroRNA-25 and in the Corresponding Exosomes}

Transfection of pre-miR-25 significantly increased miR25 levels both in MSCs and in their exosomes compared with those in MSCs without transfection and in their exosomes $(P<.001)$ (Figure 2). Transfection of control vectors did not affect the expression of miR-25 in MSCs and in their exosomes.

\section{Expressions of MicroRNA-25, NADPH Oxidase 2, and NADPH Oxidase 4 in Spinal Cords}

The expression of miR-25 could be detected in spinal cords of sham rats and increased slightly in the control group after reperfusion ( $P>.005$, vs the sham group). miR-25-enriched exosomes robustly enhanced the level of miR-25 in spinal cords $(P<.001$ vs the control, Exo, and vector-Exo groups) (Figure 3, $A$ ).

Expression of NOX2 increased significantly in the control, Exo, and vector-Exo groups, and the miR-25-Exo group after reperfusion $(P=.021, P=.016, P=.007$, and $P=.002$, respectively, vs the sham group) (Figure 3 , $B)$. No significant difference in the expression of NOX2 was observed among the 4 groups that had spinal cord ischemia $(P>.05)$.

The expression of NOX4 was also markedly enhanced in the control, Exo, and vector-Exo groups $(P=.001$, $P=.003$, and $P=.007$, respectively, vs the sham group) (Figure 3, C). However, the expression of NOX4 in the miR-25-Exo group was lower than in the control group and Exo group $(P=.002$ and $P=.012$, respectively).

\section{Interleukin-1 $\beta$ and Tumor Necrosis Factor- $\alpha$ Levels in Spinal Cords}

Transient ischemia significantly increased levels of IL- $1 \beta$ and TNF- $\alpha$ in the spinal cords of control rats $(P<.001$, vs the sham group) (Figure 4, A). Compared with the control group, levels of IL-1 $\beta$ and TNF- $\alpha$ in the Exo, vector-Exo, and miR-25-Exo groups were lower $(P<.001)$. There was no significant difference in levels of IL- $1 \beta$ and TNF- $\alpha$ in the Exo, vector-Exo, and miR-25-Exo groups $(P>.05)$.

\section{Malondialdehyde Level and Superoxide Dismutase Activity in Spinal Cords}

During reperfusion, MDA contents in spinal cords significantly increased in all the 4 groups that had spinal cord ischemia $(P<.001, P<.001, P<.001$, and $P=.010$, vs the sham group, respectively) (Figure 5, A). Although MDA levels in the Exo and vector-Exo groups were lower than in the control group, the differences did not reach statistical significance $(P=.233$ and $P=.324$, respectively). The MDA level in the miR-25-Exo group was lower than in 

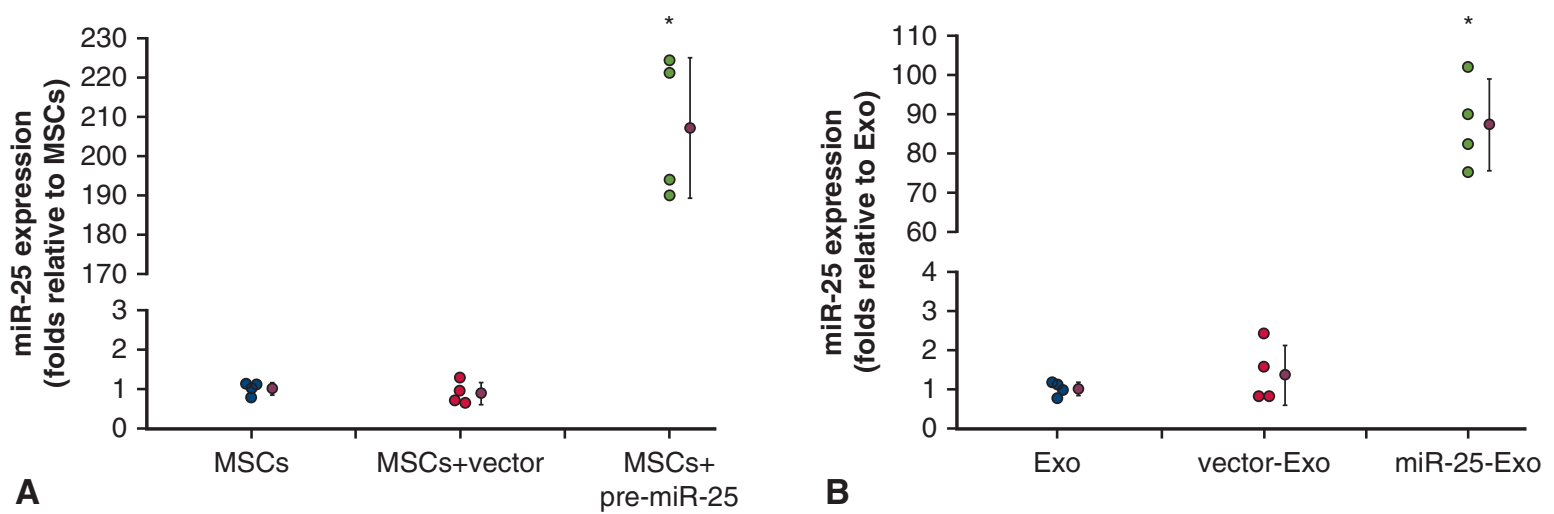

FIGURE 2. miR-25 levels in cultured MSCs (A) and the corresponding exosomes (B). ${ }^{*} P<.001$ versus MSCs or Exo. miR-25-Exo, miR-25-enriched exosomes derived from MSCs transfected with pre-miR-25; MSC, mesenchymal stem cell; Exo, exosomes derived from MSCs; vector-Exo, exosomes derived from MSCs transfected with control vectors.

the control group and Exo group $(P<.001$ and $P=.022$, respectively).

The activity of SOD remarkably decreased in the 4 groups receiving spinal cord ischemia $(P<.001$, vs the sham group) (Figure $5, B$ ). Only the activity of SOD in the miR-25-Exo group, but not in the Exo group, was significantly higher than in the control group $(P=.003)$.

\section{Neurologic Assessment}

A total of 55 male rats were enrolled in the protocol for motor function evaluation. Five rats did not survive for 7 days after transient spinal cord ischemia. Complete data were obtained in the remaining 50 rats $(\mathrm{n}=10$ for each group). A 15-minute spinal cord ischemia-induced severe neurologic injury in the control group at the 3 observation points after reperfusion manifested as higher MDI scores $(P<.001$, vs the sham group) (Figure 6 and Video 1$)$. Compared with the control group, MDI scores of the Exo, vector-Exo, and miR-25-Exo groups were significantly lower at the 3 observation points $(P<.001)$. At 48 hours and 7 days after reperfusion, MDI scores of the miR-25Exo group were even lower than those of the Exo group $(P=.003$ and $P=.004$, respectively).

\section{Histologic Examination}

Representative sections of lumbar spinal cords stained with Nissl dye are shown in Figure 7, A, and the results of counting intact motor neurons are summarized in Figure 7, $B$. Severe neurologic damage of spinal cords characterized by vacuolization, frank necrosis, and loss of motor

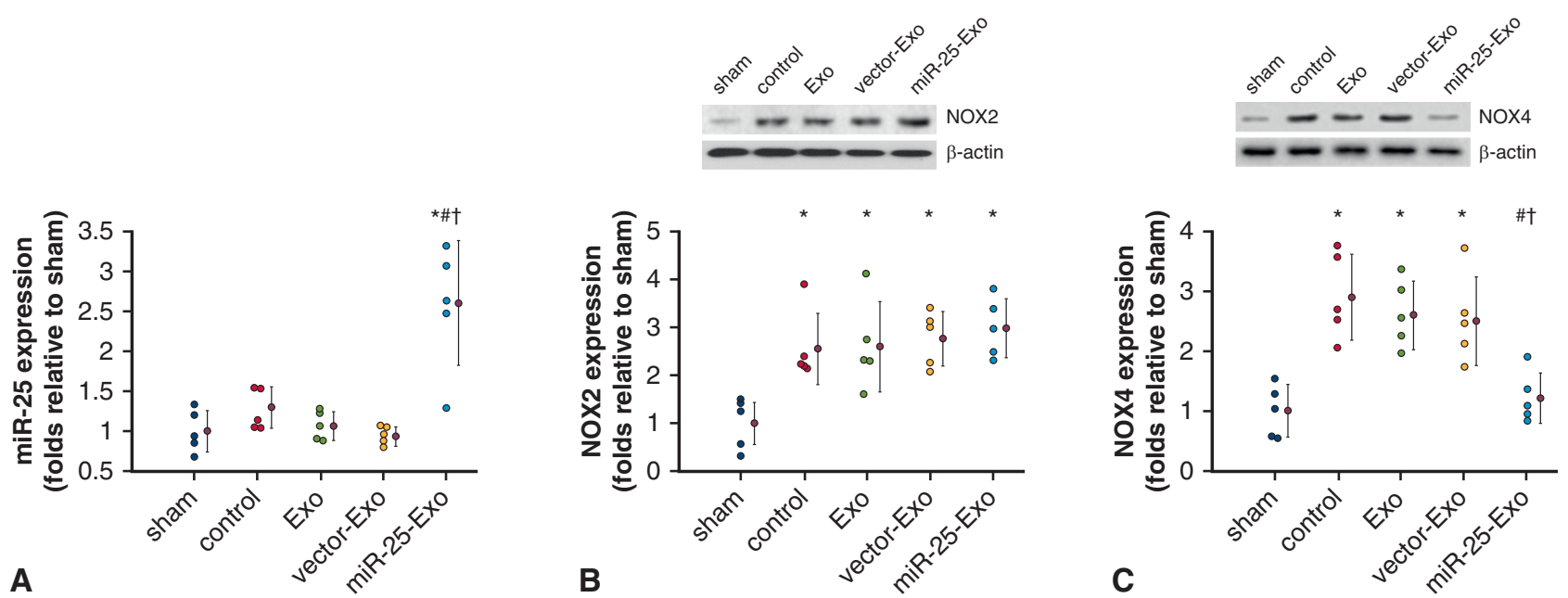

FIGURE 3. Expressions of miR-25, NOX2 protein, and NOX4 protein in spinal cords. A, miR-25 expression. B, Representative Western blots showing NOX2 expression and densitometric quantification of NOX2 expression. C, Representative Western blots showing NOX4 expression and densitometric quantification of NOX4 expression. ${ }^{*} P<.05$ versus the sham group; $\# P<.05$ versus the control group; $\dagger P<.05$ versus the Exo group. miR-25-Exo, miR-25-enriched exosomes derived from MSCs transfected with pre-miR-25; Exo, exosomes derived from MSCs; vector-Exo, exosomes derived from MSCs transfected with control vectors. 

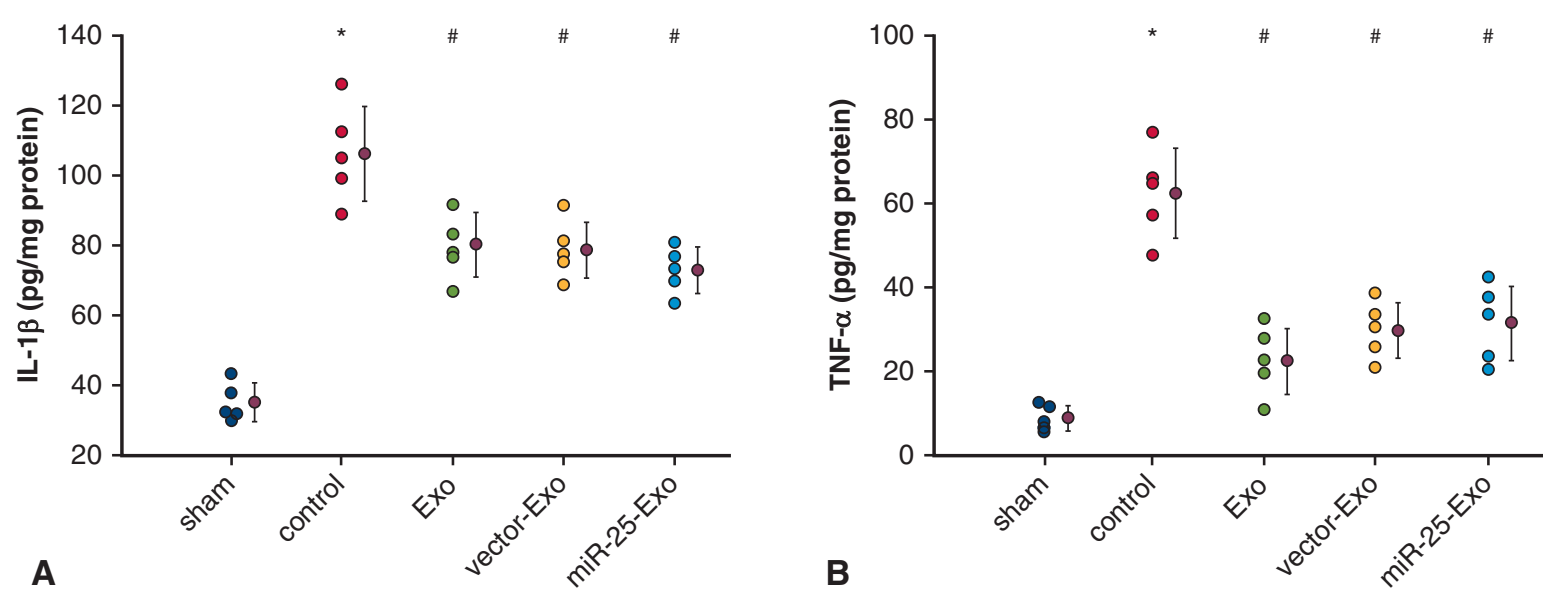

FIGURE 4. The inflammatory response in spinal cords at reperfusion. A, IL- $1 \beta$ levels in spinal cords. B, TNF-a levels in spinal cords. * $P<.001$ versus the sham group; $\# P<.001$ versus the control group. Exo, Exosomes derived from MSCs; vector-Exo, exosomes derived from MSCs transfected with control vectors; miR-25-Exo, miR-25-enriched exosomes derived from MSCs transfected with pre-miR-25.

neurons was detected in the control group. Slighter histologic changes were found in lumbar spinal cords of animals in the Exo, vector-Exo, and miR-25-Exo groups. Compared with the control group, numbers of intact motor neurons in the Exo, vector-Exo, and miR-25-Exo groups were greater $(P<.001)$. The number of intact motor neurons in the miR-25-Exo group was even greater than in the Exo group $(P=.002)$.

\section{DISCUSSION}

The salient findings of the current study are summarized in Figure 8. miR-25-enriched exosomes were derived from MSCs that were genetically modified to overexpress miR-25. Exosomes from MSCs conducted neuroprotective effects against ischemia-reperfusion injury of spinal cords in a well-established rat model, and the neuroprotection was further improved by miR25-enriched exosome.

MSCs can robustly release exosomes, and there are no differences in terms of morphologic features, isolation, and storage conditions between exosomes derived from MSCs and other sources. ${ }^{22}$ Emerging data from independent laboratories indicate that exosomes released from MSCs conduct neuroprotective effects on cerebral ischemia by promoting neural plasticity, improving poststroke neuroregeneration, enhancing angiogenesis, and preventing postischemic immunosuppression. ${ }^{9,11,23}$ Furthermore, we revealed that exosomes from MSCs induced neuroprotective effects on ischemic spinal cords as evidenced by improvement of motor function and
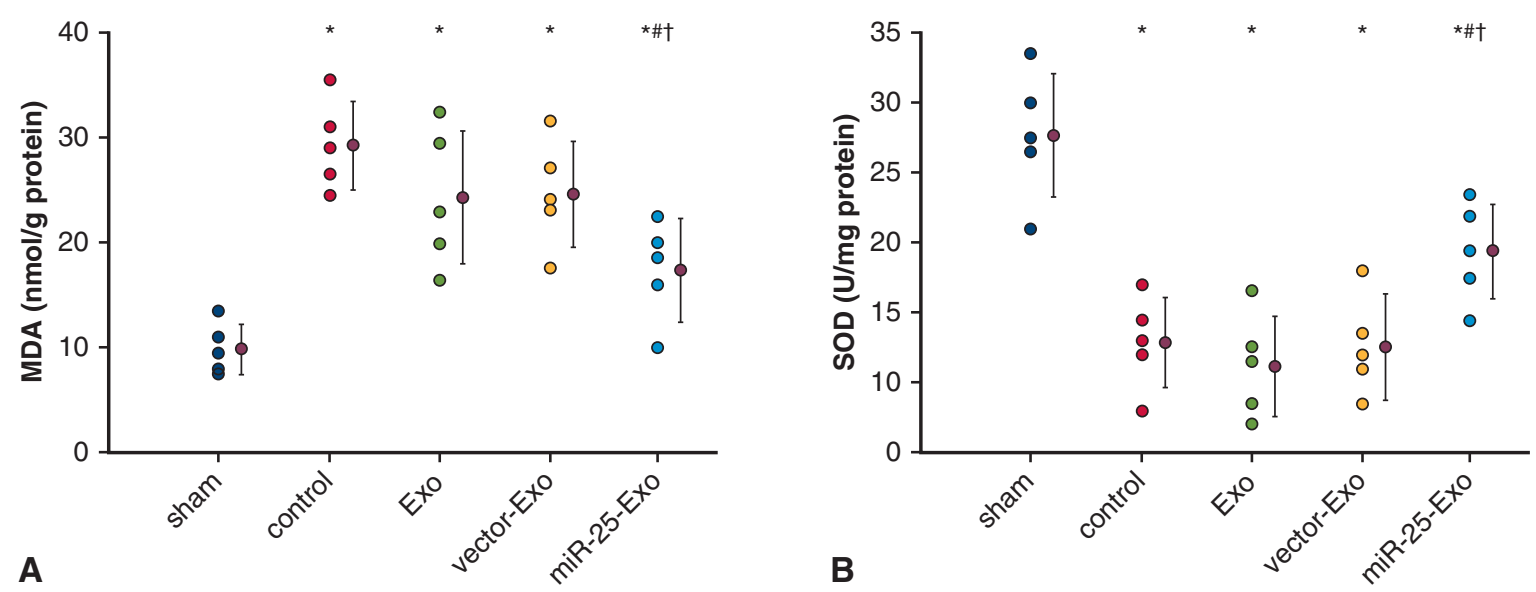

FIGURE 5. Oxidative stress in spinal cords at reperfusion. A, MDA content in spinal cords. B, SOD activity in spinal cords. $* P<.05$ versus the sham group; $\# P<.001$ versus the control group; $\nmid P<.05$ versus the Exo group. MDA, Malondialdehyde; Exo, exosomes derived from MSCs; vector-Exo, exosomes derived from MSCs transfected with control vector; miR-25-Exo, miR-25-enriched exosomes derived from MSCs transfected with pre-miR-25; SOD, superoxide dismutase. 

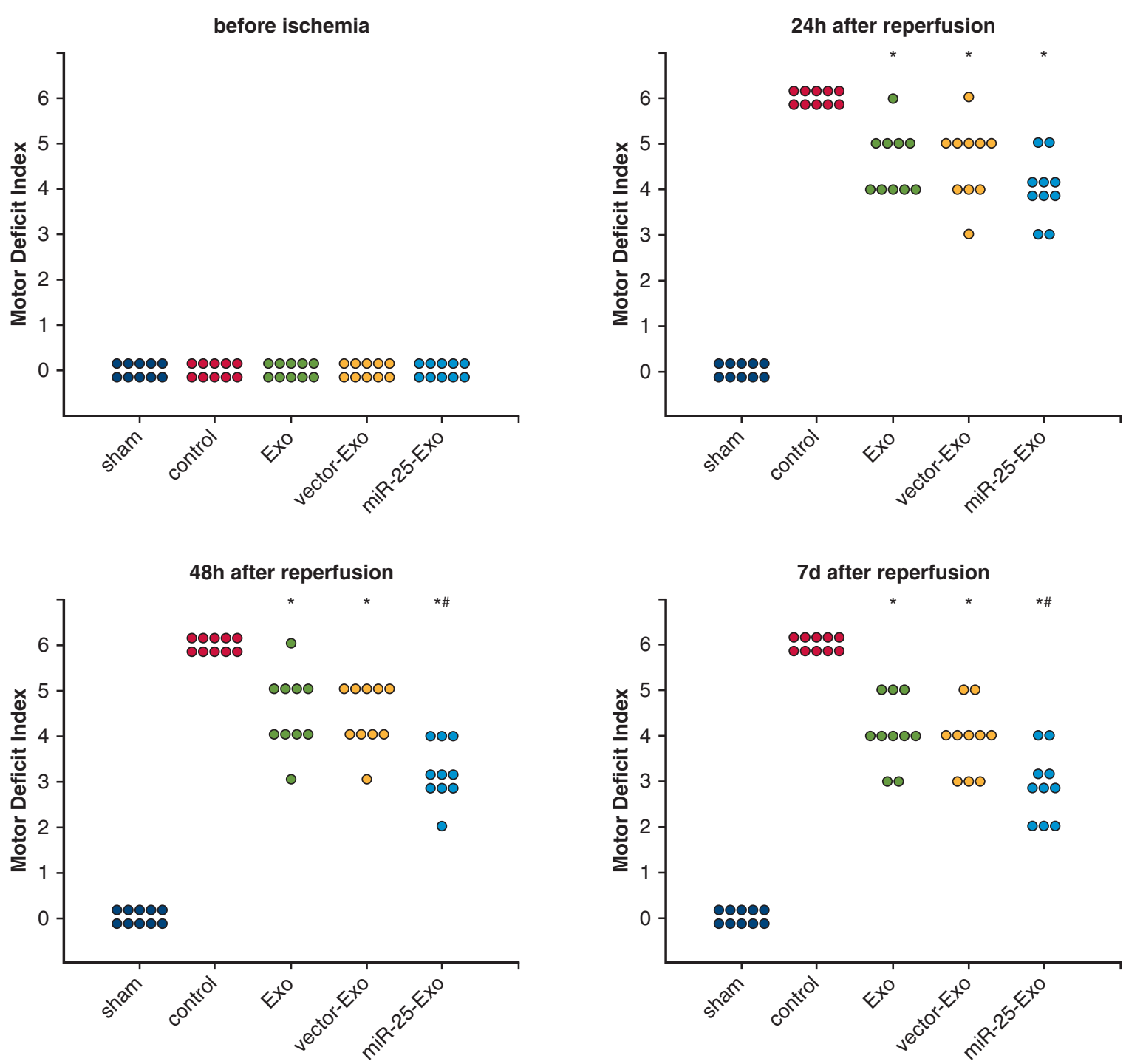

FIGURE 6. Hind-limb motor function assessment. $* P<.001$ versus the control group; $\# P<.005$ versus the Exo group. Exo, Exosomes derived from MSCs; vector-Exo, exosomes derived from MSCs transfected with control vectors; $m i R$-25-Exo, miR-25-enriched exosomes derived from MSCs transfected with pre-miR-25.

survival of motor neurons. The inflammatory response is a consequence of spinal cord ischemia and may be a major contributor to spinal cord ischemia-reperfusion injury. ${ }^{24}$ Cytokines are a group of proteins produced during activation of the inflammatory response and play an important role in the subsequent spinal cord ischemia-reperfusion injury. Both IL- $1 \beta$ and TNF- $\alpha$ are strongly expressed in the reperfusion period and remain at high levels after reperfusion. ${ }^{25}$ MSC-derived exosomes have been shown to restrain the inflammation in infarcted myocardium. ${ }^{26}$ In the current study, the elevation of IL- $1 \beta$ and TNF- $\alpha$ after reperfusion was significantly inhibited by exosomes derived from MSCs or MSCs overexpressing miR-25, suggesting that exosomes may account for the neuroprotection via anti-inflammation effects.
miRs are important functional molecules delivered by exosomes to recipient cells. By being encapsulated and contained within the exosomes, the RNA is protected from the digestion of RNAse or trypsin. ${ }^{27}$ Increasing evidence indicates that MSC-derived exosomes regulate recipient cell protein expression and modify cell characteristics through transfer of miRs. MSC-derived exosomes promote survival of retinal ganglion cells through miR-dependent mechanisms. ${ }^{28}$ MSCs communicate with brain parenchymal cells and may regulate neurite outgrowth by transfer of miR-133b to neural cells via exosomes. ${ }^{11}$ The content of exosomes can vary with cell origin and physiologic and pathologic conditions. In vitro, miR-133b levels in MSCs and in their exosomes increase after MSCs are exposed to ipsilateral ischemic tissue extracts from rats subjected to 

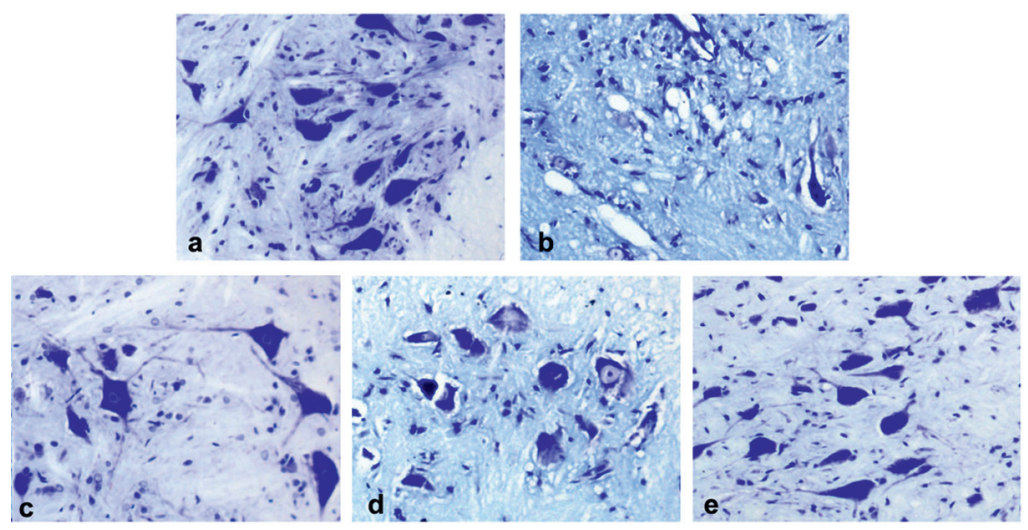

A

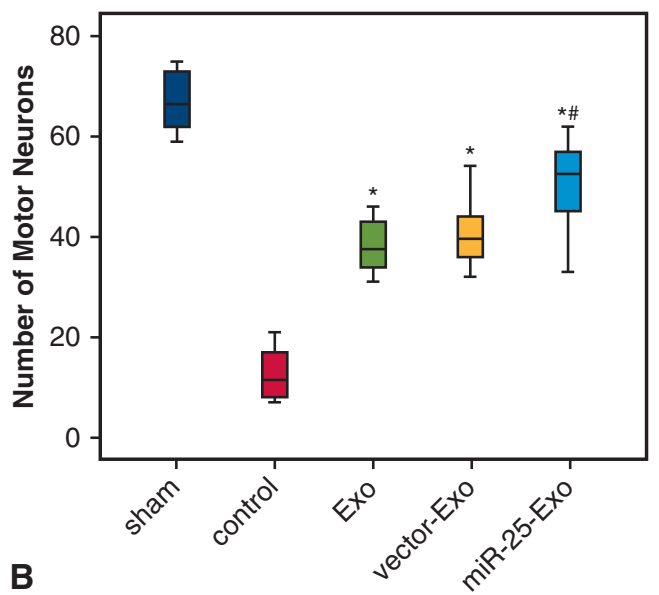

FIGURE 7. Effects of miR-25-enriched exosomes on neuron survival after spinal cord ischemia. A, Representative Nissl staining sections of spinal cords. $\mathrm{B}$, Number of motor neurons in the ventral gray matter of lumber spinal cords. ${ }^{*} P<.001$ versus the control group; $\# P=.002$ versus the Exo group. Exo, Exosomes derived from MSCs; vector-Exo, exosomes derived from MSCs transfected with control vectors; miR-25-Exo, miR-25-enriched exosomes derived from MSCs transfected with pre-miR-2.

middle cerebral artery occlusion. ${ }^{11}$ miR-126-enriched exosomes can be achieved by overexpressing miR-126 in the source adipose-derived stem cells. A better myocardial protection was induced by miR-126-enriched exosomes. ${ }^{29}$ By transfection with an miR-17-92 cluster plasmid, miR17-92 cluster-enriched exosomes are harvested and enhance
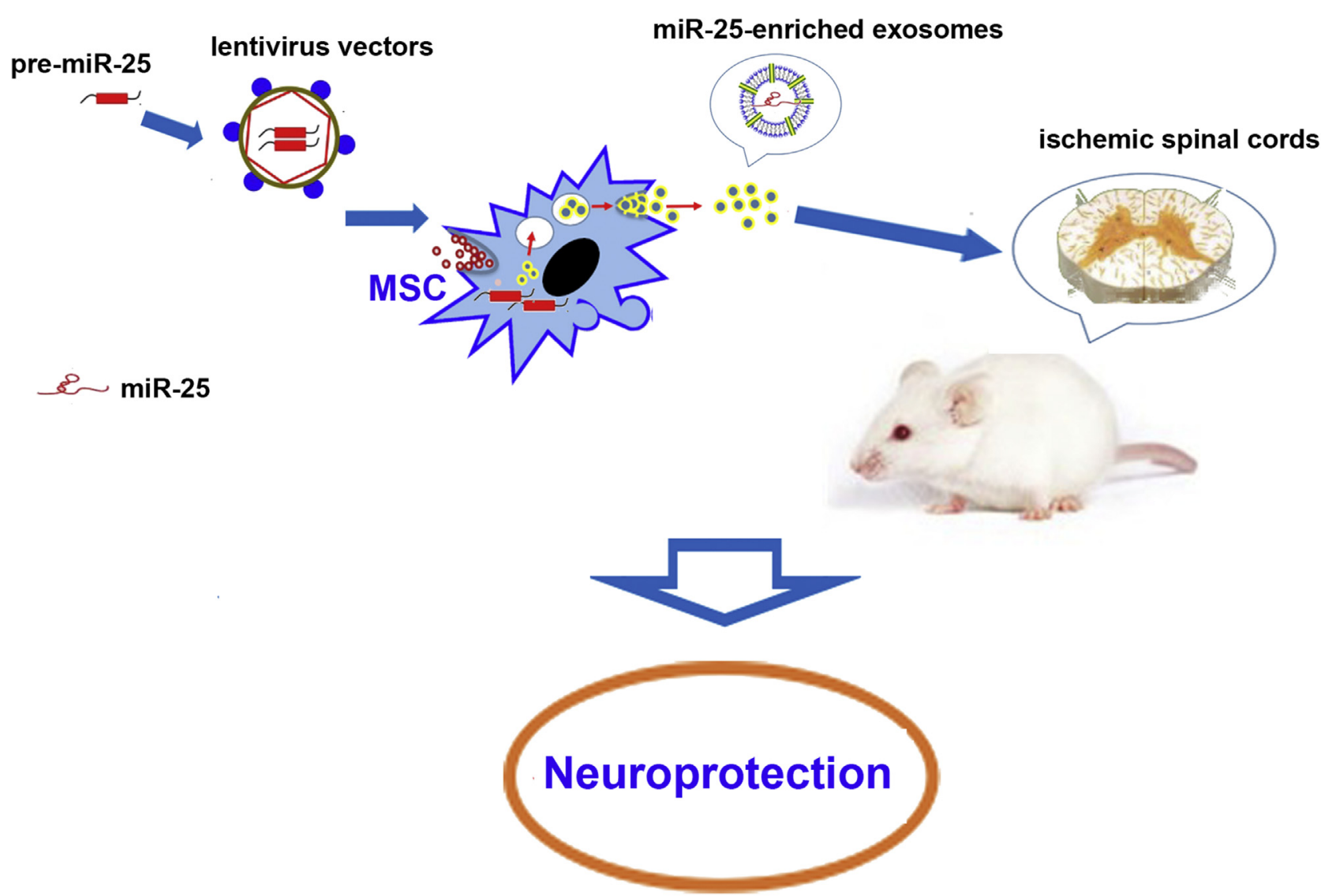

FIGURE 8. Diagram of the major findings of the current study. miR, MicroRNA; $M S C$, mesenchymal stem cell. 
neurologic recovery compared with control MSC-derived exosomes. ${ }^{30}$ All these data show that the function of exosomes from MSCs can be improved by enhancing their miR content. The expression of miR-25 was robustly increased in the exosomes from MSCs infected with lentivirus vector of pre-miR-25 in the current study. The miR25-enriched exosomes transferred miR-25 to spinal cords efficiently and provided a better neuroprotection on ischemic spinal cords compared with MSC-derived exosomes.

Reactive oxygen species (ROS) is another major mediator of the neurologic injury that follows spinal cord ischemia-reperfusion. Ischemia-reperfusion triggers an overproduction of ROS, which leads to a severe damage to cellular protein, lipid, and DNA and results in cell apoptosis and necrosis. ${ }^{31}$ Many enzymes produce ROS in the cell, but NOX is the only family of enzymes with the sole purpose of producing ROS, instead of as a by-product. $^{32}$ To date, there are 7 known NOX isoforms, of which NOX2 has been the most widely studied in the context of focal ischemia or stroke. Neuronal superoxide production contributes to cell death in both glutamate excitotoxicity and brain ischemia. NOX2 is one of the major sources of neuronal superoxide production in these settings. ${ }^{33}$ The expression of NOX2 was detected to be elevated in spinal cords after reperfusion in the current study, indicating that NOX2 was also involved in the production of ROS in ischemia-reperfusion injury of spinal cords. NOX4 is another NOX isoform related to cerebral ischemia closely. The heightened sensitivity of the brain to ischemic damage is due to an organ-specific role of NOX4 in blood-brain barrier endothelial cells and neurons. ${ }^{34}$ Mice deficient in NOX4, but not those deficient in NOX1 or NOX2, are largely protected from oxidative stress after both transient and permanent cerebral ischemia. ${ }^{35}$ The expression of NOX4 was revealed to be elevated in spinal cords after reperfusion in our study, which confirmed the essential role of NOX4 in the induction of oxidative stress in ischemiareperfusion injury of spinal cords. Another important finding of the present study was that miR-25 was transferred into spinal cords by miR-25-enriched exosomes, and thereafter only the enhancement of NOX4, but not NOX2, was abolished. With the help of bioinformatics-based databases and the published reports, miR-25 is indicated to be an endogenous regulator of NOX4. ${ }^{20,21}$ Therefore, it is plausible that NOX4 is the target protein of miR-25 by which miR-25-enriched exosomes attenuated the oxidative stress after transient spinal cord ischemia and mediated an additional neuroprotection on spinal cords compared with MSC-derived exosomes.

\section{Study Limitations}

Exosomes derived from MSCs may contain plenty of miRs. $^{2}$ Although miR-25 was highlighted and overexpressed in exosomes by genetic modification in the current study, it is not known whether other miRs aside from miR-25 contribute to the neuroprotection. Further investigation is needed to identify whether other target proteins of miR-25, besides NOX4, are associated with the neuroprotection against spinal cord ischemia. Some evidence suggests that exosomes may promote tumor growth through angiogenesis and create chemotherapy drug resistance; therefore, the potential side effects of exosomes on oncogenesis in the current model should be evaluated with a long-term experiment. Because the current results were derived from a model of only male rats, further study is needed to investigate the possible effect of gender.

\section{CONCLUSIONS}

Exosomes derived from MSCs conduct neuroprotection on ischemic spinal cords and the neuroprotection can be enhanced by genetic modification of the exosomes to contain elevated miR-25.

\section{Conflict of Interest Statement}

Authors have nothing to disclose with regard to commercial support.

\section{References}

1. Olsson C, Thelin S, Ståhle E, Ekbom A, Granath F. Thoracic aortic aneurysm and dissection: increasing prevalence and improved outcomes reported in a nationwide population-based study of more than 14,000 cases from 1987 to 2002. Circulation. 2006;114:2611-8.

2. Zhang ZG, Chopp M. Exosomes in stroke pathogenesis and therapy. J Clin Invest. 2016;126:1190-7.

3. Xiao B, Chai Y, Lv S, Ye M, Wu M, Xie L, et al. Endothelial cell-derived exosomes protect SH-SY5Y nerve cells against ischemia/reperfusion injury. Int $J$ Mol Med. 2017;40:1201-9.

4. Guitart K, Loers G, Buck F, Bork U, Schachner M, Kleene R. Improvement of neuronal cell survival by astrocyte-derived exosomes under hypoxic and ischemic conditions depends on prion protein. Glia. 2016;64:896-910.

5. Zhang ZG, Chopp M. Neurorestorative therapies for stroke: underlying mechanisms and translation to the clinic. Lancet Neurol. 2009;8:491-500.

6. Shi E, Kazui T, Jiang X, Washiyama N, Yamashita K, Terada H, et al. Intrathecal injection of bone marrow stromal cells attenuates neurologic injury after spinal cord ischemia. Ann Thorac Surg. 2006;81:2227-33.

7. Shi E, Jiang X, Wang L, Akuzawa S, Nakajima Y, Kazui T. Intrathecal injection of hepatocyte growth factor gene-modified marrow stromal cells attenuates neurologic injury induced by transient spinal cord ischemia in rabbits. Anesthesiology. 2010;113:1109-17.

8. Yeo RW, Lai RC, Zhang B, Tan SS, Yin Y, Teh BJ, et al. Mesenchymal stem cell: an efficient mass producer of exosomes for drug delivery. Adv Drug Deliv Rev. 2013;65:336-41.

9. Xin H, Li Y, Cui Y, Yang JJ, Zhang ZG, Chopp M. Systemic administration of exosomes released from mesenchymal stromal cells promote functional recovery and neurovascular plasticity after stroke in rats. J Cereb Blood Flow Metab. 2013; 33:1711-5.

10. Xin H, Li Y, Chopp M. Exosomes/miRNAs as mediating cell-based therapy of stroke. Front Cell Neurosci. 2014;8:377.

11. Xin H, Li Y, Buller B, Katakowski M, Zhang Y, Wang X, et al. Exosome-mediated transfer of miR-133b from multipotent mesenchymal stromal cells to neural cells contributes to neurite outgrowth. Stem Cells. 2012;30:1556-64.

12. Qu Y, Zhang Q, Cai X, Li F, Ma Z, Xu M, et al. Exosomes derived from miR-1815p-modified adipose-derived mesenchymal stem cells prevent liver fibrosis via autophagy activation. J Cell Mol Med. 2017;21:2491-502.

13. Kim VN, Han J, Siomi MC. Biogenesis of small RNAs in animals. Nat Rev Mol Cell Biol. 2009;10:126-39. 
14. Li G, Morris-Blanco KC, Lopez MS, Yang T, Zhao H, Vemuganti R, et al. Impact of microRNAs on ischemic stroke: from pre- to post-disease. Prog Neurobiol. 2018;163-4:59-78.

15. Liu K, Yan L, Jiang X, Yu Y, Liu H, Gu T, et al. Acquired inhibition of microRNA-124 protects against spinal cord ischemia-reperfusion injury partially through a mitophagy-dependent pathway. J Thorac Cardiovasc Surg. 2017;154: 1498-508.

16. He F, Ren Y, Shi E, Liu K, Yan L, Jiang X. Overexpression of microRNA-21 protects spinal cords against transient ischemia. J Thorac Cardiovasc Surg. 2016; 152:1602-8.

17. Jeyaseelan K, Lim KY, Armugam A. MicroRNA expression in the blood and brain of rats subjected to transient focal ischemia by middle cerebral artery occlusion. Stroke. 2008;39:959-66.

18. Guo F, Han X, Zhang J, Zhao X, Lou J, Chen H, et al. Repetitive transcranial magnetic stimulation promotes neural stem cell proliferation via the regulation of MiR-25 in a rat model of focal cerebral ischemia. PLoS One. 2014;9:e109267.

19. Zhang JF, Shi LL, Zhang L, Zhao ZH, Liang F, Xu X, et al. MicroRNA-25 negatively regulates cerebral ischemia/reperfusion injury-Induced cell apoptosis through Fas/FasL pathway. J Mol Neurosci. 2016;58:507-16.

20. Varga ZV, Kupai K, Szücs G, Gáspár R, Pálóczi J, Faragó N, et al. MicroRNA-25dependent up-regulation of NADPH oxidase 4 (NOX4) mediates hypercholesterolemia-induced oxidative/nitrative stress and subsequent dysfunction in the heart. J Mol Cell Cardiol. 2013;62:111-21.

21. Fu Y, Zhang Y, Wang Z, Wang L, Wei X, Zhang B, et al. Regulation of NADPH oxidase activity is associated with miRNA-25-mediated NOX4 expression in experimental diabetic nephropathy. Am J Nephrol. 2010;32:581-9.

22. Haraszti RA, Didiot MC, Sapp E, Leszyk J, Shaffer SA, Rockwell HE, et al, High-resolution proteomic and lipidomic analysis of exosomes and microvesicles from different cell sources. J Extracell Vesicles. 2016;5:32570.

23. Doeppner TR, Herz J, Görgens A, Schlechter J, Ludwig AK, Radtke S, et al. Extracellular vesicles improve post-stroke neuroregeneration and prevent postischemic immunosuppression. Stem Cells Transl Med. 2015;4:1131-43.

24. Zhu P, Li JX, Fujino M, Zhuang J, Li XK. Development and treatments of inflammatory cells and cytokines in spinal cord ischemia-reperfusion injury. Mediators Inflamm. 2013;2013:701970.

25. Nakata T, Kawachi K, Nagashima M, Yasugi T, Izutani H, Ryugo M, et al. Transient ischemia-induced paresis and complete paraplegia displayed distinct reactions of microglia and macrophages. Brain Res. 2011;1420 114-24.

26. Teng X, Chen L, Chen W, Yang J, Yang Z, Shen Z. Mesenchymal stem cellderived exosomes improve the microenvironment of infarcted myocardium contributing to angiogenesis and anti-Inflammation. Cell Physiol Biochem. 2015;37:2415-24

27. Valadi H, Ekström K, Bossios A, Sjöstrand M, Lee JJ, Lötvall JO. Exosomemediated transfer of mRNAs and microRNAs is a novel mechanism of genetic exchange between cells. Nat Cell Biol. 2007;9:654-9.

28. Mead B, Tomarev S. Bone marrow-derived mesenchymal stem cells-derived exosomes promote survival of retinal ganglion cells through miRNA-dependent mechanisms. Stem Cells Transl Med. 2017;6:1273-85.

29. Luo Q, Guo D, Liu G, Chen G, Hang M, Jin M. Exosomes from MiR-126 overexpressing Adscs are therapeutic in relieving acute myocardial ischaemic injury. Cell Physiol Biochem. 2017;44:2105-16.

30. Xin H, Katakowski M, Wang F, Qian JY, Liu XS, Ali MM, et al. MicroRNA clus ter miR-17-92 cluster in exosomes enhance neuroplasticity and functional recovery after stroke in rats. Stroke. 2017;48:747-53.

31. Xia Z, Chen Y, Fan Q, Xue M. Oxidative stress-mediated reperfusion injury: mechanism and therapies. Oxid Med Cell Longev. 2014;2014 373081.

32. Altenhöfer S, Kleikers PW, Radermacher KA, Scheurer P, Rob Hermans JJ Schiffers P, et al. The NOX toolbox: validating the role of NADPH oxidases in physiology and disease. Cell Mol Life Sci. 2012;69:2327-43.

33. Brennan-Minnella AM, Won SJ, Swanson RA. NADPH oxidase-2: linking glucose, acidosis, and excitotoxicity in stroke. Antioxid Redox Signal. 2010;22:161-74.

34. Casas AI, Geuss E, Kleikers PWM, Mencl S, Herrmann AM, Buendia I, et al. NOX4-dependent neuronal autotoxicity and BBB breakdown explain the superior sensitivity of the brain to ischemic damage. Proc Natl Acad Sci U S A 2017:114:12315-20.

35. Kleinschnitz C, Grund H, Wingler K, Armitage ME, Jones E, Mittal M, et al Post-stroke inhibition of induced NADPH oxidase type 4 prevents oxidative stress and neurodegeneration. PLoS Biol. 2010;8(9).

Key Words: spinal cord, ischemia, mesenchymal stem cells, exosome, microRNA 